\title{
The application of the microstructured metallic grating to light emission extraction
}

\author{
GOU YuChun \& XUAN YiMin* \\ School of Energy and Power Engineering, Nanjing University of Science and Technology, Nanjing 210094, China
}

Received January 11, 2012; accepted August 27, 2012

\begin{abstract}
Microstructured metallic gratings can be used to enhance the light emission efficiency of LEDs, and the spectral radiation properties of the LEDs vary with the different metallic materials used, leading to variation of the light emission enhancement at the same wavelength for different metallic grating materials. In this paper, the finite difference time domain (FDTD) method has been used to investigate the light emission extraction enhancement of LEDs in which gratings with different metallic materials have been applied. Through analysis of the permittivity of the metals and the quality factors of the surface plasmons (SPs), we concluded that the larger the plasma frequency obtained for the metallic interband transition, then the more suitable the metals are for light emission extraction of photons with relatively short wavelengths. This is because of the abundance of free electrons in the metals with large plasma frequencies. We also found that the wavelength-dependent trends of the extraction enhancement resulting from the scattering mechanism for different metallic materials are similar to each other. For SP-induced enhancement, either the enhancement peak position or the peak value changes significantly with the different metals.
\end{abstract}

microstructured metallic grating, LED, near-field evanescent wave, surface plasmons

Citation: Gou Y C, Xuan Y M. The application of the microstructured metallic grating to light emission extraction. Chin Sci Bull, 2013, 58: 696-700, doi: $10.1007 / \mathrm{s} 11434-012-5518-\mathrm{z}$

Control methods using the spectral properties of microstructures can be used to tune the radiative properties of light-emitting devices (LEDs). When these microstructures are introduced into the devices, the near-field radiative electromagnetic properties of the devices are changed, which can lead to an improvement in the light emission efficiency. Because of the differences between the refractive indices of the semiconductor layers in the LED chip, the photons produced by radiative recombination in the relatively high index layer suffer from total internal reflection (TIR) $[1,2]$. The evanescent waves and guided modes that result from the TIR cannot escape from the layers, which results in a reduction in the light emission efficiency.

Over the last few years, a variety of methods based on microstructures have been proposed to enhance the light emission efficiency of LEDs. These methods can be divided into two categories [2]: those that change the propagation

*Corresponding author (email: ymxuan@mail.njust.edu.cn) paths of the radiated photons through the introduction of microstructures, and those that use the microstructures to change the spontaneous emission properties of the active region of the LED chip. A method based on the surface plasmons (SPs) of the microstructures can improve the light emission efficiency attributed to the enhancement of the spontaneous emission. The SPs result from the electromagnetically stimulated collective oscillations of free electrons in the materials. Metal materials are therefore usually applied for the excitation of SPs because of the abundance of free electrons in metals. Many researchers have reported that the light emission efficiency can be enhanced by using metallic microstructures. Okamoto et al. [3] found that because of the coupling of SPs with quantum wells (QWs), a 14-fold enhancement in the peak photoluminescence intensity was observed in Ag-coated InGaN QWs, while the luminescence intensity integrated over the emission spectrum was increased by 17 times. When $\mathrm{Ag}$ nanoparticles were embedded in the semiconductor layers of InGaN/GaN mul- 
tiple QW (MQW) blue LEDs, the optical output power of these LEDs was enhanced by $32.2 \%$ [4]. An electroluminescence intensity enhancement of 1.8 times has also been observed for a GaN-based light-emitting diode with $\mathrm{Au}$ nanoparticle-arrayed layer [5]. Drezet et al. [6] found that $\mathrm{Au}$ plasmonic gratings on a high index substrate $(\mathrm{GaP})$ can enlarge the escape cone to increase the light extraction efficiency of LEDs. Because of the localized SPs on the metallic nanoparticles, at $900 \mathrm{~nm}$, a factor of 8 enhancement in the electroluminescence of a silicon-on-insulator LED with Ag nanoparticles has been demonstrated [7]. In these applications for metal nanoparticles, $\mathrm{Ag}, \mathrm{Au}$ and $\mathrm{Al}$ are most popular. However, there are few systematic studies of the application of gratings with different metal materials to light emission enhancement.

In this paper, based on our previous work, and using the finite difference time domain (FDTD) method, the effects of microstructured metallic gratings composed of the metals titanium (Ti), copper $(\mathrm{Cu})$, gold $(\mathrm{Au})$, silver $(\mathrm{Ag})$, aluminum $(\mathrm{Al})$ and the perfect electric conductor (PEC) on the light extraction efficiency of LEDs have been investigated. Through an analysis of the dielectric function of these metals and their corresponding light extraction enhancements, the practical aspects of the different metals in the different bands have been summarized. This can play a guiding role for experimental work.

\section{LED model}

Figure 1 shows a schematic of the flip-chip LED model used in the FDTD simulation. The model consists of two dielectric layers and the microstructured metallic grating. One of the dielectric layers is made of $\mathrm{GaN}$, which is used to simulate the active layer of the GaN-LED chip, and its refractive index is 2.5 [8]. In the middle of the GaN layer, MQWs are also considered, and the refractive index and thickness for each well are 2.5+i0.045 [9] and $100 \mathrm{~nm}$, respectively. The TE or TM polarized radiation source is located in the MQWs. A sapphire layer is used as the substrate, with a refractive index of 1.76 [8]. The period, filling factor, Metallic grating thickness, and the distance between the grating and the GaN layer are represented by $\Lambda, f, d$, and $h$, respectively, in which, the filling factor $f=L / \Lambda . t_{\mathrm{GaN}}$ and $t_{\text {sapphire }}$ denote the thicknesses of the GaN and sapphire layers, respectively. The chip is surrounded by an absorbing boundary condition of a perfect matched layer (PML) in the FDTD simulations. The dash dotted lines $S_{1}$ and $S_{2}$ represent the energy collecting surfaces at distances of $t_{\text {up }}$ and $t_{\text {low }}$ from the chip surfaces, respectively. In the simulation, the data collected by these two surfaces are used to calculate the enhancement factor of the light extraction. Beside the thicknesses of the PML with 20 grids, the side lengths of the calculation domain are $L_{x}$ and $L_{y}$ along the $x$-axis and $y$-axis, respectively.

To enhance the light extraction efficiency, the plasmonic grating is placed near the interface between the GaN and the sapphire layers, which is based on the coupling of the near-field evanescent wave to the propagation wave using the micro-structured grating. Because of the excitation of SPs around the metal material, the enhancement becomes more obvious.

\section{The Lorentz-Drude model of metallic dielec- tric function}

According to the features of the FDTD simulation, if the electromagnetic properties of a wavelength band are to be attained, the dielectric function that changes with wavelength is needed. The dielectric properties of metals consist of two parts: the first part is the intraband transition, which is called the free-electron model (or Drude model) $\varepsilon_{\mathrm{r}}^{\mathrm{f}}(\omega)$; the other part is the interband transition, which is called the bound-electron model (or Lorentz model) $\varepsilon_{\mathrm{r}}^{\mathrm{b}}(\omega)$. Thus, the Lorentz-Drude model of metallic dielectric function is expressed as follows [10]:

$$
\begin{gathered}
\varepsilon_{\mathrm{r}}(\omega)=\varepsilon_{\mathrm{r}}^{\mathrm{f}}(\omega)+\varepsilon_{\mathrm{r}}^{\mathrm{b}}(\omega), \\
\varepsilon_{\mathrm{r}}^{\mathrm{f}}(\omega)=1-\frac{\Omega_{\mathrm{p}}^{2}}{\omega\left(\omega-\mathrm{i} \Gamma_{0}\right)}, \\
\varepsilon_{\mathrm{r}}^{\mathrm{b}}(\omega)=\sum_{j=1}^{\mathrm{N}} \frac{f_{j} \omega_{\mathrm{p}}^{2}}{\left(\omega_{j}^{2}-\omega^{2}\right)+\mathbf{i} \omega \Gamma_{j}} .
\end{gathered}
$$

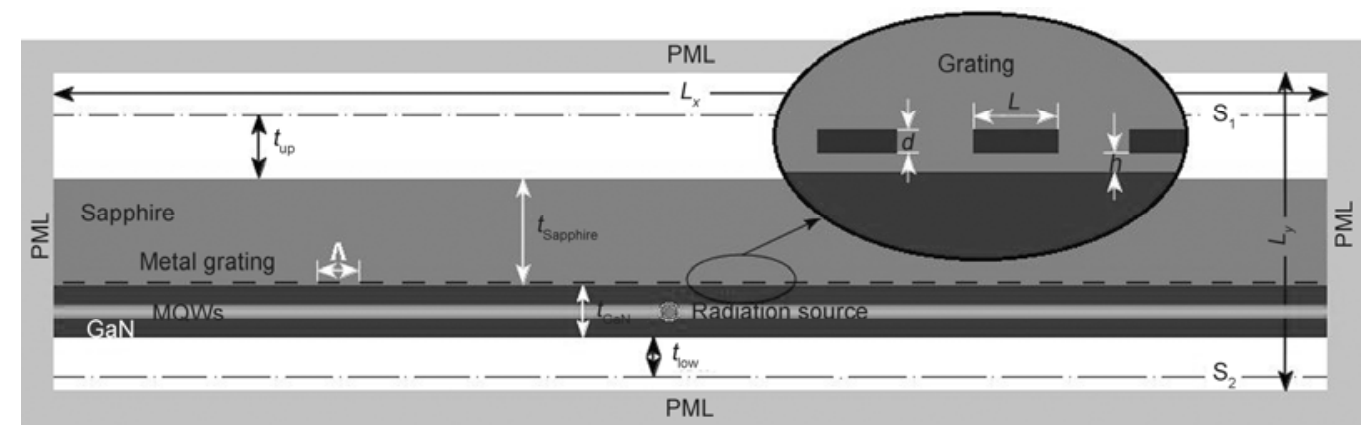

Figure 1 Schematic of the flip-chip LED model and the parameters of the structure. 
By combining eqs. (1)-(3), eq. (4) can be obtained:

$$
\varepsilon_{\mathrm{r}}(\omega)=1+\sum_{j=0}^{N} \frac{f_{j} \omega_{\mathrm{p}}^{2}}{\left(\omega_{j}^{2}-\omega^{2}\right)+\mathbf{i} \omega \Gamma_{j}},
$$

where $\omega_{\mathrm{P}}, N, \omega_{j}, f_{j}$ and $\Gamma_{j}$ represent the plasma frequency, the number of the Lorentz vibrator, the resonance frequency, the strength factor and the damping frequency, respectively. $\Omega_{\mathrm{p}}=\sqrt{f_{0}} \omega_{\mathrm{p}}$ is the plasma frequency corresponding to the intraband transition.

As a necessary condition of excitation of the SPs, the real part of the dielectric function of the material must be negative. Because abundant free electrons are present in the metal, which leads to the metal having a negative real part of its dielectric function, the metal is the best choice of plasmonic material. However, for different metals, because of the differences in the density of the free electrons, the dielectric parameters, including $\omega_{\mathrm{P}}$ and $\Gamma_{j}$, are different for each of the metals. These changes make the excitation bands of the SPs and the attenuation lengths of the SPs different. Meanwhile, the excitation of SPs also relates to the structural features of the metals and of the surrounding media. Different micro-structural features can also result in a shift in the SP band. In this paper, by using fixed dimensions for each structural parameter, different metals have been used as the plasmonic metals to analyze the applicability of each of these metals to different bands.

\section{Results and discussions}

By comparing the plasma frequencies $\Omega_{\mathrm{p}}$ of the different metals, $\mathrm{Ti}, \mathrm{Cu}, \mathrm{Au}, \mathrm{Ag}, \mathrm{Al}$ and the PEC have been selected as the plasmonic materials for the microstructured gratings. The structural parameters of the LED model are listed in Table 1.

The enhancement factor $\left(e f_{\lambda}\right)$ is defined as

$$
e f_{\lambda}=\frac{P_{\lambda, \text { with }}}{P_{\lambda, \text { without }}},
$$

where $P_{\lambda \text {,with }}$ and $P_{\lambda \text {,without }}$ represent the total energy of a specific wavelength on the energy collecting surface $S$ when the LED chip is modeled with and without metallic gratings, respectively.

Figure 2 shows the enhancement factors of the energy collecting surface $S_{1}$. The enhancement factors have been calculated for differently polarized incident waves (TM and TE waves) and the different metals. It can be shown from the figure that because of the introduction of the micro-structured metallic grating, the light extraction efficiency has been effectively improved at most wavelengths, regardless of whether the radiation source is TM or TE polarized. The mechanisms of this improvement are attributed to two sources [11]: the scattering function of the microstructured metallic grating and the excitation of SPs at
Table 1 Structural parameters of the LED model

\begin{tabular}{cc}
\hline Parameters & Values $(\mu \mathrm{m})$ \\
\hline$L_{x}$ & 3 \\
$L_{y}$ & 14 \\
$t_{\text {up }}$ & 0.5 \\
$t_{\text {low }}$ & 0.4 \\
$t_{\text {GaN }}$ & 0.5 \\
$t_{\text {Sapphire }}$ & 1 \\
$\Lambda$ & 0.5 \\
$L$ & 0.05 \\
$d$ & 0.02 \\
$h$ & 0.01 \\
\hline
\end{tabular}

the interface between the metal and the surrounding media. The enhancement trends corresponding to the scattering function are the same for all of the metals. In particular, for the TE polarized radiation source, which cannot stimulate SPs at the metal-dielectric material interface, it can be shown from Figure 2(c) and (d) that although the values of the enhancement factors vary with the metals, the position of the peak value remains the same. However, the enhancement factors resulting from the SPs vary greatly for the different metals. From the enhancement factors of the TM polarized waves, it can be shown that the peak values or the peak positions undergo significant changes if the enhancement is caused by the excitation of SPs (relatively long wavelength band).

Figure 2(b) and (d) show that the scattering function of PEC is strongest. For both TM and TE waves, the enhancement factors of PEC that result from the scattering function are the largest. This is attributed to the fact that the photons cannot enter the PEC material. Thus, the photons cannot be absorbed and are scattered totally by the material, which obviously leads to the greater enhancement. However, in the relatively long wavelength band, the enhancement factors of PEC are smaller than those of several other metals, which is because SPs cannot be stimulated using PEC materials. The enhancement for PEC is therefore based on the strong scattering of photons.

Figure 3 shows the real and imaginary parts of the dielectric functions for the five kinds of metals, which are computed using the Lorentz-Drude model. The figure indicates that the real part (absolute value) of the dielectric function of $\mathrm{Al}$ is the largest among the metals, which leads to very strong scattering of photons. With increasing wavelength, the imaginary part of the dielectric function of $\mathrm{Al}$ increases rapidly. This can result in strong absorption of photons. The dielectric function of Ti has a small real part and a large imaginary part so that photons can easily enter $\mathrm{Ti}$ and are strongly absorbed. It is shown in Figure 2 that the enhancement of $\mathrm{Ti}$ is weak, especially in the relatively long wavelength part of the visible spectrum. Because of the similar dielectric properties of $\mathrm{Cu}$ and $\mathrm{Au}$, the trends and values of the enhancement factors of the two metals are similar. In the visible spectrum, the photon absorption of $\mathrm{Ag}$ 

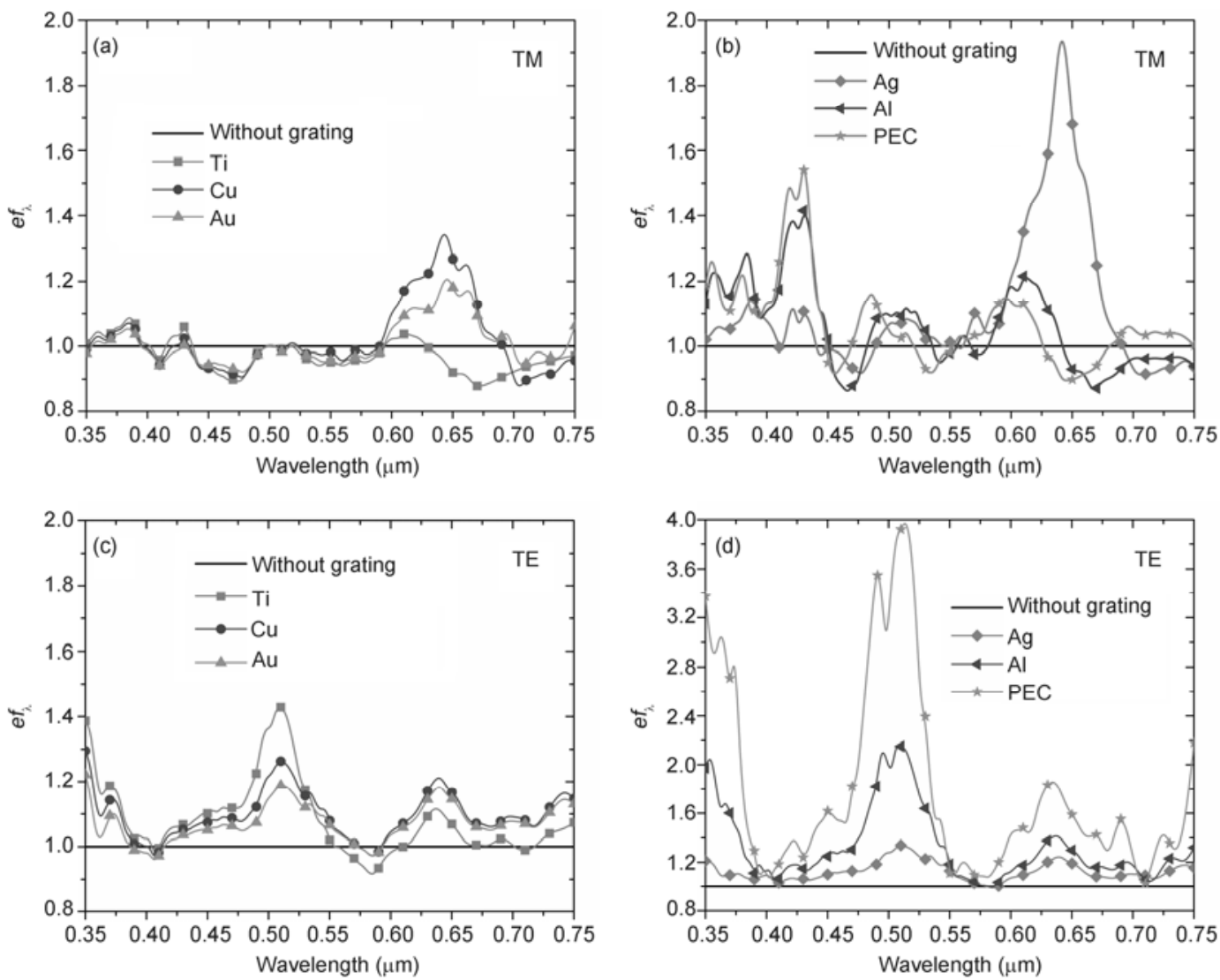

Figure 2 Enhancement factors of energy collecting surface $\mathrm{S}_{1}$ when the radiation sources are TM (p polarized) and TE (s polarized) waves.

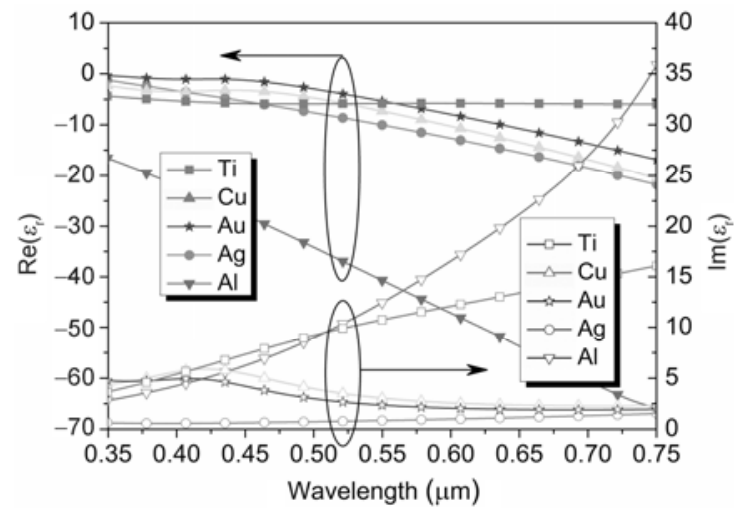

Figure 3 Changes in dielectric constant of metals with wavelength.

is weak because the imaginary part of the $\mathrm{Ag}$ dielectric function is very small $(<1.6)$. However, for the real parts of the dielectric functions of $\mathrm{Ag}$ and $\mathrm{Al}$, because the value for $\mathrm{Ag}$ is smaller than that for $\mathrm{Al}$ in the relatively short wavelength part of the visible spectrum, the $\mathrm{Al}$ grating scatters photons more strongly than the Ag grating. This is illustrated in Figure 2, where the enhancement factors corresponding to $\mathrm{Ag}$ are the smallest in the short visible spectrum for TE waves.

As long as the metal material has a negative real part of its dielectric constant, SPs can potentially be stimulated by the TM wave. The quality factor of the excited SPs varies with the different metals and wavelengths. The quality factor takes into account the real and imaginary parts of the dielectric constant, which allows it to evaluate the overall performance of the material, and it is defined as follows [12]:

$$
Q_{\mathrm{SPs}}=\frac{\operatorname{Re}\left(\varepsilon_{\mathrm{r}}\right)^{2}}{\operatorname{Im}\left(\varepsilon_{\mathrm{r}}\right)} .
$$

Figure 4 shows the quality factors of the five metals. The larger the quality factor becomes, then the stronger the SPs that are excited at the metal-dielectric interface and the weaker the damping of the photons that occurs. The figure indicates that the quality factors of $\mathrm{Ag}$ are the largest in the long visible spectrum, which results in a large enhancement factor for LED chips with microstructured Ag gratings.

From the above analysis, it can be seen that because of the differences in the metallic dielectric properties, different metals should be used in the appropriate wavelength bands to enhance light emission efficiency. For the five metals studied, i.e. $\mathrm{Al}, \mathrm{Ag}, \mathrm{Cu}, \mathrm{Au}$ and $\mathrm{Ti}$, the plasma frequencies $\hbar \Omega_{\mathrm{p}}$ are $10.83,8.28,8.21,7.87$ and $2.81 \mathrm{eV}$, respectively.

The plasma frequency of $\mathrm{Al}$ is the largest. It can be concluded that the larger the plasma frequency of the metal, then the more appropriate it is to use the metal to enhance light emission efficiency at relatively short wavelengths. It 


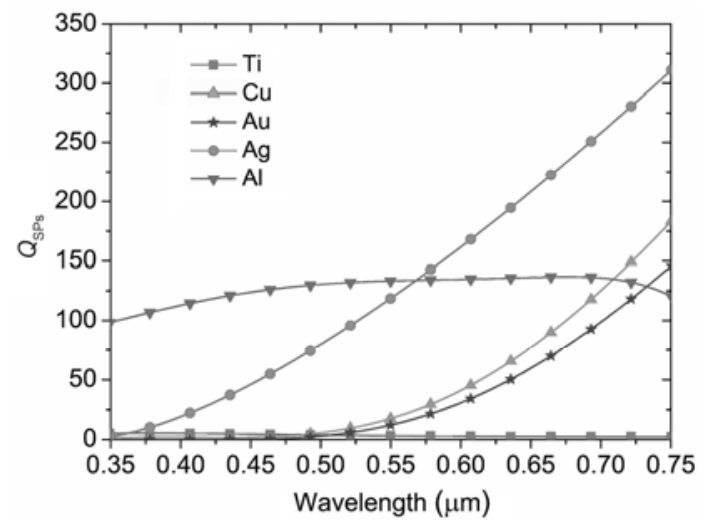

Figure 4 Quality factors of SPs for Ti, Cu, Au, Ag, and Al metals.

should be noted that because the smallest photon absorption is that of $\mathrm{Ag}$, it is thus the best plasmonic material for the visible spectrum, especially in the long wavelength band. $\mathrm{Cu}$ and $\mathrm{Au}$ are suitable for the extraction of near-infrared light. From the experimental research in the literature [13], it was also found that when the total thickness is fixed with the changes occurring in the $\mathrm{Au} / \mathrm{Ag}$ thickness ratios $(0 / 50$, 2/48, 4/46, 6/44, 8/42, 10/40, 12/38, 50/0), thicker Au layers led to longer peak wavelengths for the light emission enhancement. The wavelength corresponding to Ti should also be much longer.

\section{Conclusions}

In this paper, the effects of different metallic gratings on the light extraction efficiency of LED chips have been investigated. The enhancement factors of the light extraction efficiency are attained based on the FDTD method combined with the Lorentz-Drude model of the metal materials. The results indicate that the enhancements resulting from the scattering functions of the gratings have the same trends for different metals. For the SP-enhanced extraction, the trends and peak values of the enhancement factors depend on the metallic dielectric properties. From analysis of the metallic dielectric properties and the quality factors of the SPs, it was concluded that because of the abundance of free electrons in the metal materials with large plasma frequencies, the metal materials are closer to the PEC and are more suitable for the extraction of short wavelengths. Al is most suitable for the extraction of visible short wavelengths. Ag is an excellent SP material, and has greater advantages at visible long wavelengths. $\mathrm{Cu}$ and $\mathrm{Au}$ are more suitable for near-infrared extraction. Ti has the lowest plasma frequency, and the micro-structured $\mathrm{Ti}$ grating is not suitable for the extraction of visible light.

This work was supported by the National Natural Science Foundation of China (50936002).

1 Wang T, Guo X, Fang Y, et al. Roughening surface morphology on free-standing GaN membrane with laser lift-off technique. Chin Sci Bull, 2007, 52: 1001-1005

2 Zhmakin A I. Enhancement of light extraction from light emitting diodes. Phys Rep, 2011, 498: 189-241

3 Okamoto K, Niki I, Shvartser A, et al. Surface-plasmon-enhanced light emitters based on InGaN quantum wells. Nat Mater, 2004, 3: 601-605

4 Kwon M K, Kim J Y, Kim B H, et al. Surface-plasmon-enhanced Light-Emitting Diodes. Adv Mater, 2008, 20: 1253-1257

5 Sung J H, Kim B S, Choi C H, et al. Enhanced luminescence of GaN-based light-emitting diode with a localized surface plasmon resonance. Microelectron Eng, 2009, 86: 1120-1123

6 Drezet A, Przybilla F, Laux E, et al. Opening the light extraction cone of high index substrates with plasmonic gratings: Light emitting diode applications. Appl Phys Lett, 2009, 95: 021101

7 Catchpole K R, Pillai S. Surface plasmons for enhanced silicon light-emitting diodes and solar cells. J Lumin, 2006, 121: 315-318

8 Chao $\mathrm{C} \mathrm{H}$, Chuang S L, Wu T L. Theoretical demonstration of enhancement of light extraction of flip-chip GaN light-emitting diodes with photonic crystals. Appl Phys Lett, 2006, 89: 091116

9 Kim S K, Cho H K, Bae D K, et al. Efficient GaN slab vertical light-emitting diode covered with a patterned high-index layer. Appl Phys Lett, 2008, 92: 241118

10 Rakic A D, Djurišic A B, Elazar J M, et al. Optical properties of metallic films for vertical-cavity optoelectronic devices. Appl Opt, 1998, 37: 5271-5283

11 Gou Y, Xuan Y, Han Y, et al. Enhancement of light emitting efficiency by using combined plasmonic Ag grating and dielectric grating. J Lumin, 2011, 131: 2382-2386

12 Blaber M G, Arnold M D, Ford M J. A review of the optical properties of alloys and intermetallics for plasmonics. J Phys: Condens Matter, 2010, 22: 143201

13 Zhao H, Zhang J, Liu G, et al. Surface plasmon dispersion engineering via double-metallic $\mathrm{Au} / \mathrm{Ag}$ layers for III-nitride based lightemitting diodes. Appl Phys Lett, 2011, 98: 151115

Open Access This article is distributed under the terms of the Creative Commons Attribution License which permits any use, distribution, and reproduction in any medium, provided the original author(s) and source are credited. 